\title{
Uso de Pesquisa Bibliográfica em Informática na Edu- cacão: um Mapeamento Sistemático
}

\author{
The use of Literature Review in Informatics in Education: a Systematic Mapping
}

\author{
Juliana Patrícia Detroz \\ Universidade do Estado de Santa Catarina (UDESC) - \\ Programa de Pós Graduação em Computação Aplicada \\ (PPGCA) \\ julidetroz@gmail.com
}

\author{
Marcelo da Silva Hounsell \\ Universidade do Estado de Santa Catarina (UDESC) - \\ Programa de Pós Graduação em Computação Aplicada \\ (PPGCA) \\ marcelo.hounsell@udesc.br
}

\author{
Mauro Hinz \\ Universidade do Estado de Santa Catarina (UDESC) - \\ Programa de Pós Graduação em Computação Aplicada \\ (PPGCA) \\ mmhinz@gmail.com
}

Resumo Uma Pesquisa Bibliográfica (PB) é um procedimento que visa identificar pesquisas já publicadas, o estado da arte, tendências ou lacunas sobre determinado tema com vistas na extensão do conhecimento científico. Existem, pelo menos, 4 métodos de PB: tradicional, bibliométrica, mapeamento e, revisão. Quando executadas de forma sistemática as PBs apresentam maior rigor científico, confiabilidade, imparcialidade e reproducibilidade, apesar de exigirem a adoção e registro de quantidade maior de passos durante sua realização. Este artigo identificou quantos e quais métodos de PB estão sendo utilizados na área de Informática na Educação no Brasil e avaliou como estão sendo conduzidos. Para tal, foi executado um mapeamento sistemático das publicações nacionais da área nos últimos 10 anos. Um total de 3149 artigos oriundos de 4 revistas e 4 eventos da área no Brasil foram avaliados resultando na identificação de 17 mapeamentos ou revisões sistemáticas e 25 PBs tradicionais ou bibliométricas, mesmo que não fosse evidente pelos seus titulos. Uma análise quantitativa foi realizada sobre as 42 PBs e uma síntese qualitativa das 17 sistemáticas. Os resultados indicam que o número PBs vem aumentando como um todo, em especial as sistemáticas, mas a PB tradicional ainda é a mais frequente. Foi evidenciada uma proliferação de termos usados para identificar artigos de PB, podendo ser encontrados em várias sub-áreas. Os dados também indicam que há uma falta de uniformidade e rigor nos procedimentos e até desacordo com a terminologia (alguns artigos se autodenominaram erroneamente, conforme os critérios desta pesquisa) a ponto de dificultar pesquisas terciárias como esta. Concluímos que a comunidade científica de Informática na Educação se beneficiaria de (i) maior rigor na execução de PBs e, (ii) da concentração da produção nacional num único portal com recursos de busca. Estas medidas certamente facilitariam o desenvolvimento da área como um todo no Brasil.

Palavras-Chave: Metodologia da Pesquisa, Pesquisa Bibliográfica, Informática na Educação

\footnotetext{
Abstract Literature review $(L R)$ is a procedure that aims at identifying published research, state of the art, trends or gaps in a particular field towards extending the scientific knowledge. There are four methods for performing LRs: Traditional; Bibliometric; Reviews, and; Mappings. When performed through a systematic procedure, LRs are more scientifically rigorous, reliable, unbiased and reproducible although requiring the adoption of more steps and registering while performing the search. This paper investigate how many and which LR methods are being used in the field of Informatics in Education in Brazil and how they have been performed. A systematic
} 
mapping has been performed over the last 10 year of publications in Brazil. A total of 3149 papers from 4 journals and 4 proceedings have been scanned producing a total of 17 papers identified as systematic, regardless their title, and 25 tradicional or bibliometric LRs. A quantitative analysis was performed considering all $42 \mathrm{LRs}$ and a qualitative analysis over the 17 systematic publications. Results show that the number of LRs has been growing, particularly systematic ones, but traditional LR still is the most frequent one. It was found that a wide variety of terms have been used to refer to LRs and they have been applied to many sub-areas. Data also show a lack of procedures' rigors and uniformity as well as disagreement to the terminology (some papers identify themselves differently from the criteria used here) that made it difficult to perform tertiary researches like this. We conclude that the Informatics in Education scientific community could certainly benefit from (i) a more rigorous use of LRs and, (ii) putting together all national papers produced in the field in a single portal with search capabilities. These measures would help develop the field further in Brazil.

Keywords: Research Methodology, Literature Review, Informatics in Education

\section{Introdução}

O A primeira etapa no processo de investigação cientifica usualmente é uma Pesquisa Bibliográfica (PB), sendo esta a responsável pela busca de referenciais teóricos, trabalhos relacionados e até do 'estado da arte' em um tema específico com vistas ao reconhecimento da fronteira do conhecimento. Conforme Wazlawick [1], apesar de não produzir conhecimento novo, este tipo de pesquisa é de fundamental importância para a realização de estudos científicos, servindo como fonte de informações para aumentar o domínio de conhecimento do pesquisador sobre a área de estudo".

Ainda segundo Wazlawick [1], a PB é definida como o estudo de artigos, teses, livros e outros tipos de publicações disponibilizadas por editoras e indexadas. Já para Lakatos e Marconi [2] a PB "trata-se de levantamento de toda a bibliografia já publicada, em forma de livros, revistas, publicações avulsas e imprensa escrita." Estas autoras (op. cit.) complementam que "sua finalidade [da PB] é colocar o pesquisador em contato direto com tudo aquilo que foi escrito sobre determinado assunto, com objetivo de permitir ao cientista o reforço paralelo na análise de suas pesquisas ou manipulação de suas informações".

Petersen et al. [3] afirmam que, como consequência da expansão e evolução de uma área de pesquisa há um aumento no número de publicações e resultados obtidos. Desta forma, torna-se importante sintetizar este conhecimento, a fim de apresentar uma visão geral da área estudada. Esta assertiva reafirma a necessidade da execução de uma PB, que tem como objetivo, apresentar o estado da arte da área de estudo em questão, podendo também apontar lacunas a serem exploradas.

Porém, a condução e/ou apresentação de uma PB de forma incompleta pode levar a resultados tendenciosos (biased), impossibilitando a replicação dos métodos adotados e até mesmo conduzir a conclusões incorretas.

O rigor científico é vítima da simplificação que os processos digitais trazem ao automatizar a publicação de artigos e como consequência há um aumento no número de publicações e resultados obtidos, levando a mais quantidade e mais dificuldade de garantir a qualidade de uma PB diante de todo material disponível na internet. Por isto, passa a ser cada vez mais importante o uso de métodos mais rigorosos e eficientes aplicados a serem aplicados à própria $\mathrm{PB}$.

Existem vários tipos de PB: a PB tradicional, as revisões sistemáticas de literatura, os mapeamentos sistemáticos de literatura e as bibliométricas.

Para auxiliar na execução de $\mathrm{PB}$, pode-se fazer uso das ferramentas de busca acadêmicas na internet (ASE, do inglês Academic Search Engine), que facilitam a pesquisa por publicações de artigos em periódicos e conferências. Porém, a disponibilidade de um ferramental valioso não soluciona o problema, sendo necessário o uso de métodos específicos para tirar o melhor proveito da ferramenta.

Alguns autores apontam para o aumento de publicações de PBs, os chamados estudos secundários, não só na medicina $[4,5]$, mas também na área de engenharia de software e da computação de forma geral [6]. Os estudos primários caracterizam-se por apresentarem relatos de descobertas científicas, como resultado, por exemplo, de experimentos ou estudos de caso. Uma pesquisa secundária realiza a revisão de vários estudos primários relevantes a um tema específico, com objetivo de sintetizar as evidências já identificadas nesta área. Um estudo terciário executa uma revisão dos artigos secundários relacionados a um mesmo tema [7]. 
Estas observações, somadas ao fato da Informática na Educação já estar inserida no âmbito educacional há mais de trinta anos, como discutido por Valente [8] em um trabalho que talvez tenha sido a primeira PB brasileira publicada na área, serviram como fator motivacional da presente pesquisa. Portanto, para que a comunidade científica de Informática na Educação possa se beneficiar dos métodos de PB, é importante identificar como ela tem executado este processo para depois identificar como melhorá-lo.

Este artigo tem como objetivo identificar quantos e quais métodos de PB estão sendo utilizados pela comunidade científica brasileira de Informática na Educação, em especial as revisões e mapeamentos, e investigar como estes estão sendo conduzidos de forma a garantir sua imparcialidade e replicabilidade. Para isto, faz-se relevante realizar um alinhamento dos principais termos e procedimentos que caracterizam cada tipo de $\mathrm{PB}$, de forma a garantir um melhor entendimento e maior clareza dos critérios adotados neste levantamento. A fim de delimitar o escopo do estudo, foram considerados apenas os artigos publicados nos últimos 10 anos, ou seja, no período compreendido entre os anos de 2003 e 2012 nos principais veículos (revistas ou eventos) de publicação da área no Brasil.

O presente trabalho encontra-se organizado da seguinte maneira: na seção 2 são apresentadas definições dos tipos de PB e suas características. A seção 3 descreve de forma sucinta trabalhos relacionados a esta pesquisa. Na seção 4 é detalhado o planejamento e condução do levantamento dos trabalhos. A seção 5 contém a síntese narrativa dos artigos analisados. Na seção 6 são apresentados os resultados obtidos e em 7 são apresentadas as observações identificadas a partir da seção anterior sendo então, na seção 8 apresentadas algumas considerações finais.

\section{Pesquisa bibliográfica sistemática}

Os métodos de Pesquisa Bibliográfica (PB) sistemáticas surgiram na medicina $\mathrm{e}$ foram adotados por várias áreas de estudo, como por exemplo, as Ciências Sociais, Economia e Engenharia de Software [9]. Sutton e Higgins [5] afirmam que o método de meta-análise é utilizado na medicina há mais de 100 anos e que nos últimos 30 , o impacto deste tipo de estudo vem crescendo consideravelmente. Na medicina, a importância das revisões sistemáticas é vista com tamanha relevância que em 1993 foi fundada a The Cochrane Collaboration, uma instituição responsável pela organização, manutenção e disseminação de revisões sistemáticas, sendo estas preparadas por voluntários e autores conceituados que trabalham em um de seus 52 grupos de pesquisa [10].

$\mathrm{Na}$ Ciência da Computação, o uso do paradigma ba- seado em evidências para realização de revisões sistemáticas começou a ser discutido na Engenharia de Software após a publicação do estudo de Kitchenham e Dybå [11]. Neste processo são definidas várias etapas que visam atingir o objetivo da pesquisa de forma imparcial e garantindo que a mesma possa ser auditável ou replicável por outros pesquisadores.

\section{As PB podem ser classificadas em:}

- Tradicional: que não possuem um processo sistemático definido, sendo orientadas pela experiência do pesquisador. Neste tipo de pesquisa não há garantia de que os resultados sejam imparciais, podendo estes enfatizar os dados que corroboram com as hipóteses apoiadas pelo pesquisador;

- Bibliométrica: A bibliometria é definida por Pritchard [12] como "a aplicação de métodos estatísticos e matemáticos para obtenção de informação quantitativa sobre livros e outros meios de comunicação". Neste tipo de PB são obtidos dados como, por exemplo, a relevância de determinado artigo ou da influência de determinado pesquisador a partir no número de citações feitas a este trabalho. Vale ressaltar que a pesquisa bibliométrica, apesar de não ser chamada de sistemática, adota um procedimento inicial semelhante a estas;

- Revisões sistemáticas de literatura (SLR, do inglês, Sistematic Literature Review): que visam identificar e avaliar de forma confiável e imparcial todas as pesquisas relevantes para um tema, apresentando de forma sintetizada seus principais conceitos e resultados obtidos [7]. A condução da revisão sistemática visa identificar e reportar todas as pesquisas relevantes à área e não apenas aquelas que corroborem a hipótese apoiada pelo pesquisador. São denominados usualmente como survey, review, levantamento ou até panorama;

- Mapeamentos sistemáticos da literatura (SLM Sistematic Literature Mapping): aplicado usualmente quando identifica-se que há pouca evidência ou que o tema abordado é bastante abrangente, oferecendo desta forma uma visão geral da área de estudo e quantificando os resultados [3]. Através desta visão, é possível identificar tendências de pesquisa ou lacunas a serem estudadas. Podem ser encontradas na literatura identificadas, entre outros, como overview ou meta-análise;

- Revisões terciárias: que, assim como a revisão deste artigo, produzem o levantamento de revisões secundárias já publicadas em uma área de estudo, podendo estas serem executadas como SLRs ou SLMs. Kitchenham e Charters [7] descrevem, no anexo 3 de sua pesquisa, a definição do protocolo do 
processo sistemático para a condução de uma revisão terciária. Petersen et al. [3] apresentam em seu trabalho um guia com orientações para a condução de mapeamentos sistemáticos.

\subsection{Mapeamento sistemático}

Os passos principais para a execução de um mapeamento sistemático, segundo Petersen et al. [3] são: a definição das perguntas da pesquisa (RQ, do inglês, Research Question), a busca por trabalhos relevantes, a seleção destes trabalhos, a extração e mapeamento dos dados. A Figura 1, mostra o esquema do processo sistemático, onde é possível visualizar cada etapa (em cima) e o resultado obtida através da mesma (em baixo).

As perguntas de pesquisa orientam a condução da pesquisa para obtenção dos dados relevantes para alcançar o seu objetivo principal. Com as RQs definidas, temse como resultado uma visão do escopo da pesquisa que

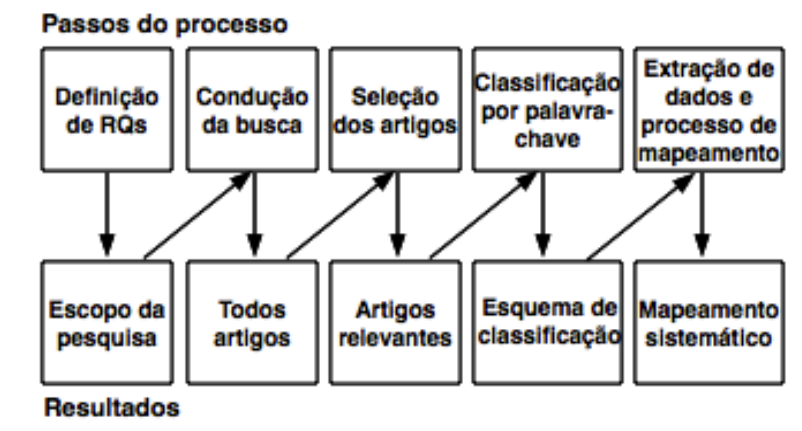

será executada.

Figura 1 : Processo de mapeamento sistemático [3]

Em seguida, faz-se a busca por estudos primários (ou secundários, caso trate-se de uma revisão terciária como a deste artigo) através da adoção de estratégias de busca, podendo esta ser: Manual: realizando a leitura dos resumos e títulos de todos os artigos de determinado veículo e identificando aqueles que contenham as palavras-chaves procuradas, e; Automatizada: definindo strings de busca que contenham composições booleanas das palavraschaves e utilizando-as em ASEs.

Após esta etapa, tem-se um total de artigos que poderão ser selecionados através da aplicação de critérios de inclusão e exclusão, a fim de excluir os trabalhos não relevantes para responder às RQs.

A partir da leitura dos artigos relevantes, define-se um esquema de classificação dos artigos, com objetivo de agrupar os trabalhos por características ou palavraschaves específicas. Estas palavras-chaves podem definir, entre outros, aspectos referentes ao tema da pesquisa, resultados e processo.
Através do esquema de classificação, é possível, na etapa de extração de dados, mapear os trabalhos de forma a obter dados estatísticos que auxiliem a responder às RQs e identificar tendências ou lacunas da área pesquisada.

\section{Trabalhos relacionados}

Alguns autores já apresentaram pesquisas com o intuito de mapear os artigos de PB em outras áreas de estudo.

Cruzes et al. [6] realizaram uma pesquisa terciária na área de Engenharia de Software que visou analisar os tipos e a utilização dos métodos de síntese de pesquisa em revisões sistemáticas. Quase metade dos 31 estudos incluídos na revisão não continha qualquer síntese; dos que fizeram, dois terços realizaram uma síntese narrativa ou temática. Os autores observaram que apesar do interesse gerado pelas revisões sistemáticas, pouca atenção ainda é dispensada à síntese de pesquisa na Engenharia de Software.

Kitchenham et al. [13] apresentaram um estudo terciário também na área de Engenharia de Software para identificar como as revisões sistemáticas estão sendo conduzidas na área. Foram encontrados, através de uma busca manual, 20 artigos relevantes a este estudo e identificaram que a qualidade das PBs aumentou com o uso do processo sistemático, apesar de grande parte dos pesquisadores ainda executarem PB tradicionais.

Hanssen et al. [14] identificaram, através de um estudo terciário, as tendências da área de Engenharia de Software global. Para a condução do processo sistemático, foram adaptadas as orientações apresentadas por Kitchenham e Charters [7], sendo os dados coletados a partir de 12 trabalhos selecionados neste processo. Os autores concluíram que desenvolvimento ágil é um tema em evidência nas pesquisas da indústria de software.

Zhang e Babar [15] realizaram uma investigação empírica sobre a adoção, relevância e uso de SLRs na área de Engenharia de Software. Os autores sugerem que o principal fator de motivação para a realização de revisões sistemáticas é a descoberta de ideias inovadoras para novas pesquisas e concluem que o SLR está tornando-se uma metodologia de pesquisa popular para a execução de PB.

A pesquisa conduzida por Imtiaz et al. [16] visou identificar as experiências de condução de revisões sistemáticas de literatura e afirmam que esta revisão beneficia os novos pesquisadores que buscam executar uma PB sistemática pela primeira vez. Como resultado, foram identificados 116 trabalhos relevantes ao tema, sendo observado que as etapas de definição de estratégia de busca, de bases de dados, planejamento e extração de 
dados são as mais desafiadoras na condução de um SLR.

Silva et al. [17] executaram a atualização do SLR realizado por Kitchenham et al. [13], com o objetivo de verificar se houve um aumento na publicação de artigos de SLR deste a execução do estudo terciário anterior. A pesquisa evidenciou a importância do processo sistemático de revisão da literatura, comprovando que, quando conduzido de forma metódica, rigorosa e sendo o processo bem documentado, a revisão sistemática pode ser confirmada por outros pesquisadores, gerando os mesmos resultados. Isto reforça o caráter científico da pesquisa em questão nos aspectos de confiabilidade e reproducibilidade. Este trabalho foi restrito à terminologia específica, o que sugere que os autores (op. cit.) consideram que os termos e procedimentos adotados já estão consolidados na área de Engenharia de Software.

Influenciado pela área da saúde, a Engenharia de Software passou a ser mais exigente na execução de PBs e com isto houve uma perceptível evolução na área, entre outros aspectos, por ter adotado um rigor na terminologia empregada. Este rigor na terminologia não foi alcançado ainda por outras áreas da ciência computação. $\mathrm{Na}$ área de Informática na Educação, por exemplo, como este trabalho irá mostrar, este rigor ainda está em desenvolvimento.

Todos os trabalhos apresentados nesta seção mostram uma tendência de aumento na adoção de PB sistemáticas bem como as vantagens desta adoção. Apesar da importância constatada, não se identificou nenhum estudo terciário sobre pesquisas sistemáticas com foco nos veículos mais importantes da área específica de Informática na Educação no Brasil.

\section{Metodologia de análise}

Esta pesquisa foi conduzida de acordo o processo de mapeamento sistemático, apresentado na seção 2.1.

\subsection{Definição das RQs}

O estudo foi conduzido de modo a responder as seguintes perguntas de pesquisa (RQ):

(a) RQ1: Quais e quantos tipos e métodos de PB estão sendo utilizadas na área de Informática na Educação no Brasil?

(b) RQ2: Como os trabalhos se autodenominam e se isso condiz com a definição do respectivo tipo de $P B ?$

(c)RQ3: Quais os elementos do protocolo de revisão sistemática estão sendo utilizados pelas pesquisas?

\subsection{Condução da busca}

Para a realização deste mapeamento sistemático, definiu-se avaliar os artigos publicados em 4 periódicos e 4 eventos científicos brasileiros que tratam do tema Informática na Educação, sendo estes:

- Simpósio Brasileiro de Informática na Educacão (SBIE);

- Workshop sobre Educação em Computação (WEI), anteriormente denominado Workshop de Ensino de Informática;

- Workshop de Informática na Escola (WIE);

- Workshop de Desafios da Computação Aplicada à Educação (DesafIE!);

- Revista Brasileira de Informática na Educação (RBIE);

- Revista de Informática Teórica e Aplicada (RITA);

- Revista Novas Tecnologias na Educação (RENOTE);

- Revista Informática na Educação: Teoria \& Prática.

Como não se identificou um site com todos os arquivos de todos os artigos aceitos nestes veículos, o processo de busca foi conduzido em duas etapas. A primeira, consistiu em uma busca manual nos periódicos e eventos que possuem um site que disponibilize todos os artigos publicados pelos veículos em questão, sendo esta pesquisa realizada com auxílio da opção de pesquisa, quando presente nos sites.

Nesta coleta foram consideradas as publicações que apresentavam em seu título ou abstract as seguintes palavras-chaves:

- REVISÃO;

- PESQUISA/REVISÃO SISTEMÁTICA;

- REVISÃO DE LITERATURA;

- PESQUISA/REVISÃO BIBLIOGRÁFICA;

- PESQUISA BIBLIOMÉTRICA;

- ESTADO DA ARTE;

- LEVANTAMENTO;

- MAPEAMENTO SISTEMÁTICO;

- MAPEAMENTO;

- META-ANÁLISE; 
- OVERVIEW;

- SURVEY;

- REVIEW;

- PANORAMA;

- PRODUÇÃO CIENTÍFICA, e;

\section{- ESTUDO BIBLIOGRÁFICO.}

$\mathrm{Na}$ segunda etapa, foram utilizadas ferramentas de busca para identificar páginas de divulgação disponibilizadas pela instituição de ensino superior responsável por organizar cada edição dos eventos WEI e DesafIE!. Desta forma, foi possível obter a lista de artigos aceitos para, então, executar a busca manual pelas palavras-chaves citadas. Para o WEI as palavras-chaves foram aplicadas apenas ao título, já que não se obteve acesso aos resumos de todos os estudos. Além disto, os anos de 2003, 2007 e 2008 não foram considerados nesta pesquisa, uma vez que não foram identificados todos os artigos aceitos nestas edições do evento. Nestas duas etapas foram analisados um total de 3149 artigos, resultando na seleção de 185 artigos.

Após a busca usando as palavras-chaves através das ferramentas de pesquisa disponibilizadas pelos veículos, foram verificados manualmente os artigos publicados nos eventos e periódicos, a fim de validar a qualidade das palavras-chaves $(\mathrm{KW})$ adotadas e identificar se algum trabalho condizente com o escopo desta pesquisa não foi obtido através das palavras utilizadas. Foram encontrados, e considerado na amostragem, mais 3 artigos que tratam de pesquisas bibliográficas, mas que não deixavam clara esta informação em seus títulos e resumos ou utilizavam denominações alternativas pouco usuais na literatura. Desta forma, isto representa que a busca pelas palavras-chaves adotadas obteve uma taxa de erro de $1,59 \%$ do número total de estudos selecionados.

\subsection{Seleção dos artigos}

Após a busca por palavras-chaves, um total de 188 artigos foram identificados, sendo estes submetidos aos critérios de inclusão (CI) e exclusão (CE), a fim de obter os estudos pertinentes para responder às perguntas da pesquisa. Os critérios definidos foram:

(i) CI1: Considerar somente artigos cientificos com- pletos (mais de 4 páginas);

(ii) CI2: Incluir artigos de pesquisas bibliográficas de qualquer tipo;

(iii) CI3: Abranger artigos os quais foi possivel obter o acesso ao seu conteúdo completo;

(iv) CE1: Eliminar artigos que não tratassem do tema Informática na Educação;

(v) CE2: Excluir artigos que tenham como objetivo principal a apresentação de ferramentas, propostas de metodologias ou discussão de temas, sendo a PB um recurso auxiliar;

(vi) CE3: Remover trabalhos que se denominam pesquisas bibliográficas, porém que mais de 50\% de suas fontes não sejam artigos científicos;

(vii) CE4: Desconsiderar artigos repetidos ou versões resumidas de artigos maiores (full ou de periódicos).

A Figura 2 apresenta o esquema do processo de coleta dos dados utilizados na aplicação desta pesquisa e o número de artigos considerado em cada etapa. $\mathrm{Na}$ parte de baixo da mesma tem-se o número de artigos resultantes, para cada tipo de PB. Esta figura enfatiza o conceito de que o processo de pesquisa bibliográfica sistemática é um filtro e executado de forma bastante laboriosa. Visualmente são apresentados todos os passos e a relação sequencial entre eles, estendendo e detalhando a figura clássica proposta por Petersen et al. [3], a qual é amplamente reproduzida em PBs sistemáticas quaisquer, e que encontra-se reproduzida na Figura 3.

Optou-se por aplicar primeiramente os critérios de exclusão (CE), sendo que caso a publicação avaliada se enquadrasse em pelo menos um destes, o mesmo não seria avaliado daí em diante.

Além disto, o artigo deveria atender a, pelo menos, um dos critérios de inclusão. Do total de 188, 18 artigos foram eliminados através do CE1 e 125, 26 e 2 pelos critérios CE2, CE3 e CE4, respectivamente. Observe que um mesmo trabalho pode ter se enquadrado em mais de um critério de exclusão, sendo contabilizado em cada um deles. 


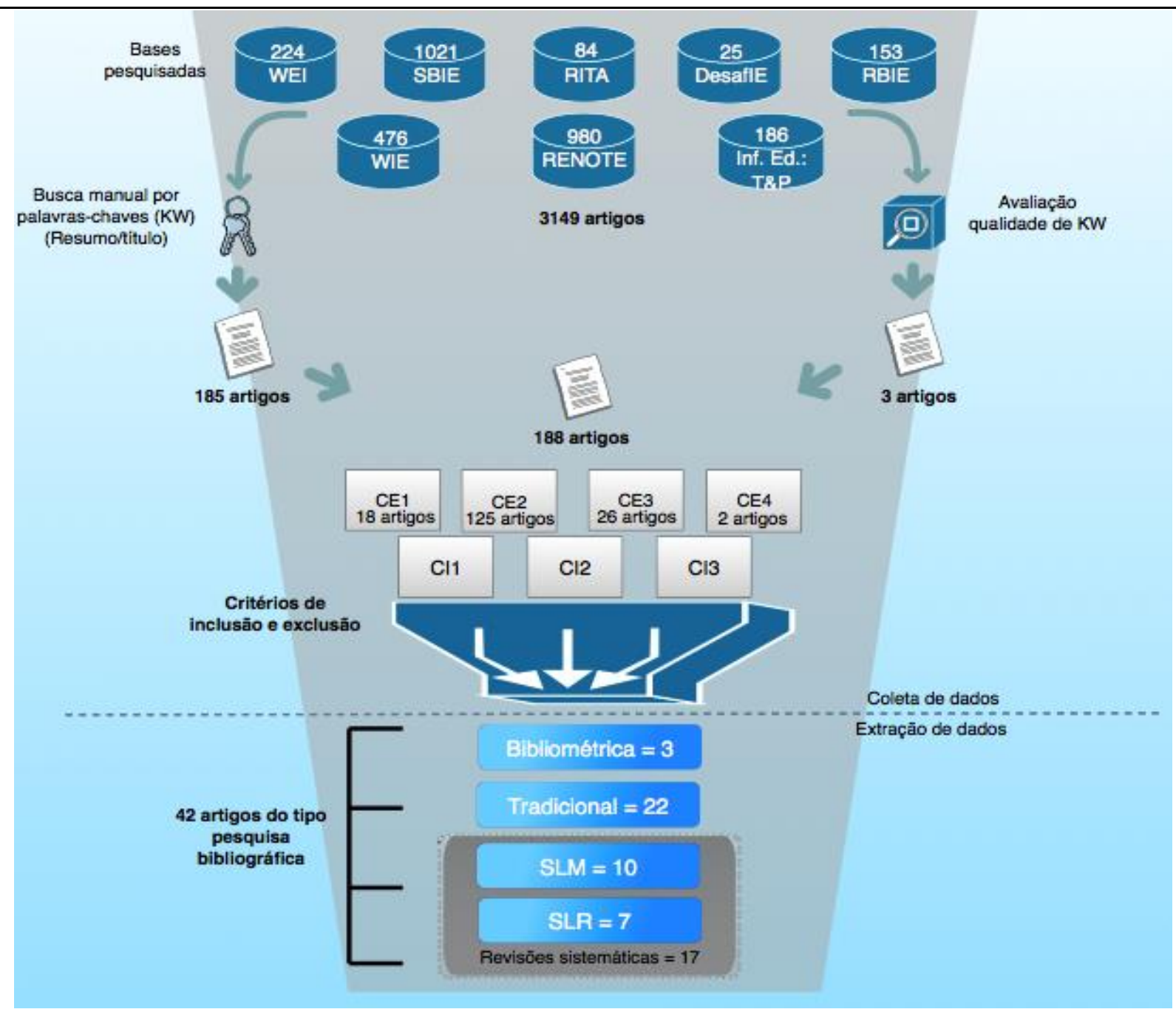

Figura 2 : Esquema do processo de coleta e extração de dados

$\mathrm{Na}$ aplicação dos CEs e CIs, assim como na extração dos dados, foi realizada a análise por dois co-autores, Detroz e Hinz, separadamente. Os resultados desta análise foram comparados e os casos de divergências revistos um a um, onde parte dos conflitos foram sanados. Os casos em que persistiram dúvidas ou conflitos foram solucionados pelo terceiro co-autor, Hounsell.

\subsection{Esquema de classificação e extração de dados}

Após a aplicação dos CIs e CEs, foram identificadas 42 PBs relevantes ao escopo desta pesquisa dentre os 188 artigos selecionados, sendo que 17 destas foram consideradas pertinentes para o escopo desta pesquisa, ou seja, classificadas como SLMs ou SLRs. O conteúdo destes trabalhos foi analisado para a execução da etapa de extração de dados, na qual se observou as características da execução de processo sistemático e o tipo de revisão sis- temática conduzida. Não foi necessário nenhum tipo de agrupamento da classificação adotada neste mapeamento. A Figura 3, apresenta o resultado da filtragem de artigos como consequencia do processo sistemático adotado nesta pesquisa.

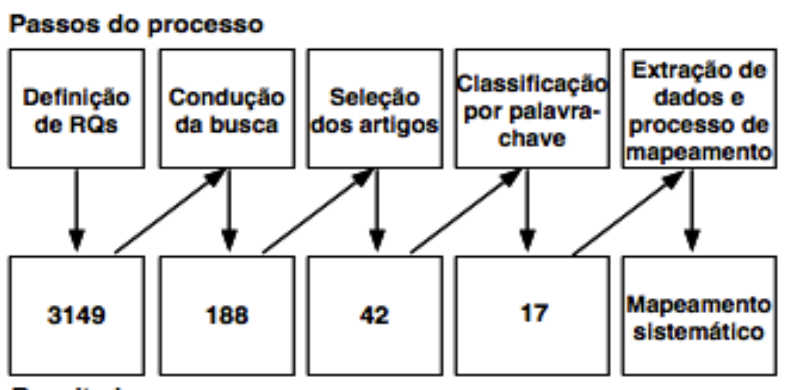

Resultados

Figura 3: Processo do mapeamento sistemático

Desta forma, as informações mapeadas de cada artigo foram: ano de publicação, veículo de publicação (perió- 
dico, evento), palavra-chave que identifica o artigo, como os autores autodenominam o trabalho (revisão sistemática, PB, entre outros), a área de estudo, se o artigo apresenta: (a) a estratégia de busca adotada (as palavraschaves usadas), (b) os critérios de inclusão(c) e exclusão, (d) as bases ou ASEs consultados, (e) a pergunta de pesquisa (RQ), (f) se define um critério para resolução de conflitos na seleção dos artigos e aplicação dos critérios de inclusão e exclusão, (g) se há síntese ou discussão dos dados coletados, (h) se a pesquisa foi conduzida por dois ou mais pesquisadores e; a partir destas informações, como os autores da presente pesquisa classificam a $\mathrm{PB}$ avaliada (SLM, SLR, Tradicional ou Bibliométrica).

Na comparação da classificação gerada pelos pesquisadores, apenas 4 artigos foram classificados como sendo do mesmo tipo pelos 2 primeiros co-autores. $\mathrm{Na}$ análise conjunta, através da qual os artigos foram avaliados novamente 7 conflitos foram solucionados. A classificação dos 6 restantes, foi definida com a orientação do terceiro co-autor.

\section{Síntese}

A seguir é apresentada uma síntese narrativa dos 17 trabalhos classificados como SLM ou SLR ordenados pelo veículo e ano de publicação. Nesta síntese são apresentadas as evidências principais dos estudos e características do processo sistemático executado.

No artigo de Barros et al. [18] é apresentada uma revisão (SLR) sobre pesquisas empíricas do uso de computadores no ensino fundamental e médio. O mesmo utiliza como referência o processo sistemático descrito na literatura médica. Para avaliar a qualidade dos artigos encontrados, foram selecionados aleatoriamente 46 do total de 377 artigos eliminados pelos CIs e CEs, sendo estes lidos por um dos autores a fim de garantir que os mesmos realmente não fazem parte do escopo da pesquisa. Através desta análise identificou-se que apenas 2 destes não deveriam ter sido eliminados, o que corresponde a uma taxa erro global de 5\%. Apesar de analisar e discutir o conteúdo dos estudos, o que o caracteriza como SLR, esta pesquisa apresenta também indícios de SLM, como a apresentação de meta-análises.

Maximo et al. [19] realizaram um mapeamento (SLM) sobre a informática e o tipo de avaliação adotado em Ensino a Distância na UFRGS durante o período entre 1998 e 2008. Os trabalhos avaliados foram coletados no servidor do domínio da universidade em questão. Apesar de apresentar as expressões de busca utilizadas e descrever que, a partir da leitura dos mais de 800 registros encontrados por esta estratégia, foram eliminados aqueles que não tratavam da avaliação dos alunos, não foram explicitados os CIs e CEs, o que dificulta a replicação do processo. Como um dos resultados da pesquisa é apresentada uma tabela com o tipo de enfoque avaliativo de cada um dos artigos. A partir deste mapeamento é realizada uma discussão e identificadas tendências.

No SLR conduzido por Igarashi et al. [20], foi levantado o alinhamento entre os estudos científicos nacionais e internacionais sobre aprendizagem organizacional e tecnologia da informação, constatando como resultado que a cultura influencia no compartilhamento do conhecimento e no destaque dado a diferentes percepções. Após o processo de coleta dos dados, é apresentada uma síntese narrativa dos trabalhos selecionados e apresentadas conclusões sobre os mesmos.

Löbler et al. [21] realizaram um SLM com o objetivo de mapear as pesquisas sobre inclusão digital aplicadas à área de administração. Como resultado os autores concluíram que, dentre os artigos selecionados, nenhum procurou mensurar o nível de inclusão social de autonomia sobre as Tecnologias de Informação, identificando assim lacunas a serem exploradas. São descritas as estratégias de busca e bases utilizadas na pesquisa, mas o texto não apresenta indícios de outros componentes do processo sistemático, como por exemplo, os CIs, CEs e de resolução de conflitos.

Pietruchinski et al. [22] conduziram em sua pesquisa um SLM sobre os jogos educativos apresentados em artigos científicos publicados no Simpósio Brasileiro de Informática na Educação (SBIE) durante o período entre 2001 e 2010. Os autores identificaram a necessidade de realizar uma revisão semelhante em outros veículos de publicação devido ao pequeno número de evidências encontradas. $\mathrm{O}$ processo sistemático foi realizado com base no procedimento proposto por [7], sendo apresentadas de forma clara as questões da pesquisa, a estratégia de busca, CIs, CEs, entre outros. Os resultados obtidos foram tabelados e apresentados de forma estatística, caracterizando-o como um mapeamento.

Através de um SLM, também baseado na proposta de [7], os autores Valaski et al. [23] levantaram os modelos de estilo de aprendizagem aplicados à adaptação e personalização dos materiais de aprendizagem. Assim, como no trabalho de Pietruchinski [19], são apresentadas as estratégias de busca, questões de pesquisa e critérios de exclusão. É feita uma breve síntese onde são apresentados os modelos de estilo de aprendizagem referenciados por cada trabalho.

Bogoni e Pinho [24] apresentaram um SLR sobre a utilização de simuladores de Endodontia baseados em Realidade Virtual e equipados com dispositivos hápticos, identificando os tipos de equipamentos e metodologias utilizadas os testes destes simuladores. A revisão utilizou os preceitos propostos por Pai [25], novamente uma referência da literatura médica. Apesar disto, a estrutura do processo sistemático é semelhante ao aplicado usualmen- 
te na área de Computação, apresentando as estratégias de busca, bases de dados, critérios de inclusão, entre outros. $\mathrm{Na}$ extração dos dados são apresentados os principais resultados de cada artigo selecionado, além de seus objetivos, metodologias aplicadas, dispositivos utilizados e conclusões.

Penteado et al. [26] conduziram um SLM com a finalidade de apresentar um estudo sobre os ambientes virtuais de aprendizagem (AVAs) enquanto base de apoio aos objetos de aprendizagem (OAs). Os artigos selecionados foram mapeados por categorias definidas pelos pesquisadores e por ano versus veículo de publicação, através do qual os autores identificaram um aumento nas publicações que tratam do tema em questão.

O SLM conduzido por Aureliano e Tedesco [27] mapeia as publicações que reportam experiências de ensino de programação para iniciantes. Esta pesquisa baseia-se no protocolo proposto por [7], apresentando seus componentes de forma estruturada e bem definida. Os resultados extraídos desta coleta foram apresentados de forma estatística. Uma característica desta pesquisa é que a mesma teve a preocupação em descrever as limitações da mesma.

O artigo de Filho e Barbosa [28] apresenta um SLR para compreender como a comunidade científica descreve e investiga as características e requisitos no novo paradigma de ensino. A partir das buscas realizadas, 438 documentos foram retornados. Após a análise, foram selecionados 198 trabalhos, que após a leitura dos resumos e a aplicação dos CIs e CEs, o número de trabalhos foi reduzido para 36. Excluindo-se os trabalhos repetidos entre as bases, 22 artigos foram selecionados ao final do processo.

Abreu et al. [29] apresentam um SLM para investigar quais tipos de tecnologias vêm sendo utilizadas para auxiliar o desenvolvimento de software educativo. Este estudo visou encontrar mecanismos para guiar o desenvolvimento de software educacional e apontar lacunas nesta área. Quatro questões foram levantadas para guiar o mapeamento sistemático e após a avaliação de um total de 1636 estudos inicialmente retornados, 65 foram selecionados como estudos relevantes para o mapeamento.

O artigo de Medeiros e Gomes [30] apresenta um SLM para investigar e reunir as abordagens para monitoramento e rastreamento das atividades e interações em ACVA, destacando o alcance e as limitações dessas abordagens. A partir das buscas realizadas, 162 documentos foram retornados. Após a análise, 48 foram selecionados como estudos relevantes para o mapeamento.

Os autores Vermelho et al. [31] conduziram um SLM sobre os resultados de pesquisas nacionais executadas no ano de 2011 sobre o tema Redes Sociais. A partir das buscas realizadas, 1.298.429 documentos foram retornados. Após a análise, 101 foram selecionados, os quais ainda foram lidos os títulos que tivessem maior aderência ao tema "Redes Sociais" + Saúde e a área de conhecimento. $\mathrm{O}$ resultado final foi uma seleção de 49 artigos.

$\mathrm{O}$ artigo de Lima et al. [32] apresenta um SLM para levantar as técnicas e os processos utilizados para desenvolver software educativo. Como resultado de busca foram identificados 724 estudos relevantes. Destes, foram excluídos alguns com base nos títulos e palavras-chave, resultando 47 estudos. Finalmente, foram obtidos 27 estudos primários, com base no resumo e conclusão dos estudos.

Paludo e Raabe [33] executaram um SLR com o objetivo de analisar jogos educativos de computador para gerência de projetos de software. A partir das buscas realizadas, 22 documentos foram retornados. Após a análise, 13 foram selecionados e comparados através de 22 critérios definidos pelos autores.

A pesquisa de Benetti e Albano [34] apresenta um SLM sobre como o Teste de Software vem sendo ensinado em cursos superiores e empresariais. A partir das buscas realizadas, 103 estudos. Após a análise, 100 foram selecionados como estudos relevantes para o mapeamento. Dos estudos mais diretamente ligados ao tema foram encontrados ao todo 36 artigos. Porém, destes, 23 não apresentavam uma avaliação do método proposto ou essa avaliação não era detalhada. Restando assim os 9 estudos escolhidos como resultado deste mapeamento.

$\mathrm{O}$ artigo de Oliveira et al. [35] apresenta um SLM com a finalidade de identificar os estudos que envolvem a prática de PBL com o ensino da Ciência da Computação. Após a avaliação inicial de 2464 artigos, foram selecionados 52 estudos primários relevantes para a pesquisa. A seleção dos 52 estudos primários foi realizada através dos critérios de inclusão e exclusão.

\section{Resultados}

Dos 188 artigos selecionados, 42 tinham como objetivo principal realizar uma pesquisa bibliográfica (PB). A Figura 4 apresenta a distribuição destes trabalhos por tipo (considerando a classificação realizada pelos autores da presente pesquisa) e ano de publicação.

A linha contínua na Figura 4 apresenta o total de artigos publicados em cada ano, sendo possível notar um aumento significativo deste tipo de pesquisa nos últimos dois anos. $\mathrm{O}$ aumento do número de pesquisas sistemáticas mostra-se recente, sendo que este crescimento foi mais expressivo no SBIE, agora evento incluso no CBIE (Congresso Brasileiro de Informática na Educação). Por ser um evento que reflete a tendência mais recente das pesquisas realizadas da área de Informática na Educação, 
entende-se que há um direcionamento da comunidade científica no sentido de adotar as PBs sistemáticas.

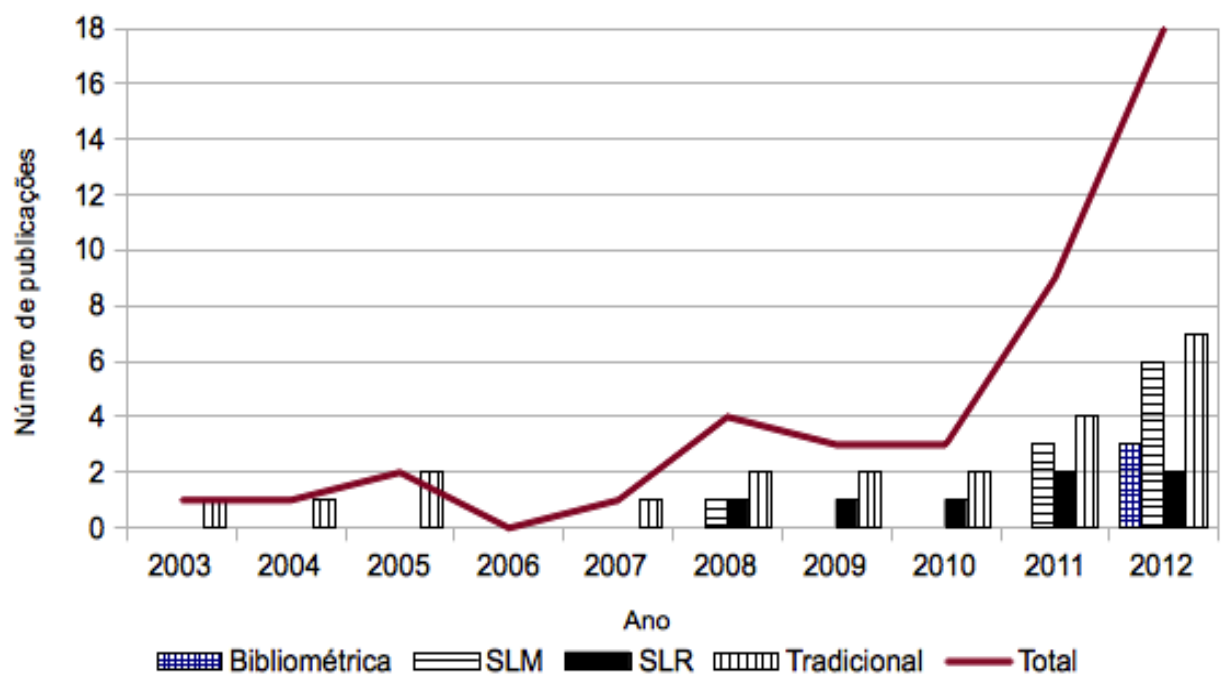

Figura 4 : Distribuição de artigos por tipo e ano de publicação

No período entre 2003 e 2012, o SBIE foi o veículo que publicou o maior número de revisões sistemáticas, tendo sido encontrados 10 artigos de SLM ou SLR em um total de 17 identificados por esta pesquisa, como mostra a Figura 5. Porém, considerando o total de pesquisas bibliográficas, e não apenas as revisões sistemáticas, o veículo que publicou o maior número de artigos deste tipo no período analisado foi a revista RENOTE.
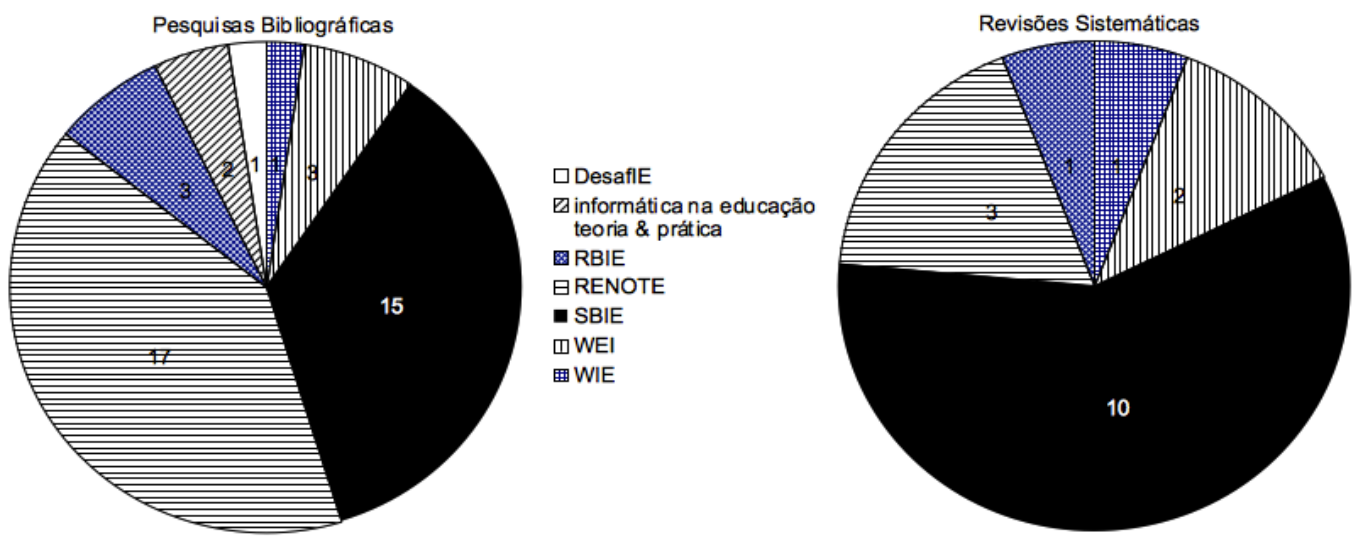

Figura 5 : Quantidade de pesquisas bibliográficas e revisões sistemáticas por veículo de publicação

De forma relativa ao total de artigos publicados em cada veículo no período de análise, a proporção de pesquisas bibliográficas apresentou-se, de forma geral, uniforme em grande parte dos eventos e periódicos, representando entre $1 \%$ e $2 \%$ das pesquisas.

A Figura 6, apresenta os percentual relativo da quantidade de PBs pelo total de artigos publicados pelos veículos. O DesafIE foi o evento com maior valor percentu- al, porém isto pode ser explicado pelo reduzido número de publicações e pelo foco do evento que intenciona analisar as tendências e maiores desafios na área. Dos 25 artigos aceitos 1 refere-se a uma $\mathrm{PB}$, alcançando 4\%. O veículo periódico com destaque na área por ser específico, a RBIE, apresentou a segunda maior média percentual e o SBIE 17 das 1021 publicações classificam-se como PB. 
Figura 8 : Distribuição de artigos por palavra-chave.

Os termos utilizados com maior frequência foram "mapeamento" e "revisão", sendo encontrados em 37 e 29 artigos, respectivamente. As palavras-chaves "mapeamento sistemático" e "overview" foram as menos utilizadas nos trabalhos avaliados, sendo identificadas em apenas um artigo cada uma.

Neste estudo foi avaliado se as pesquisas estavam utilizando as denominações de SLM e SLR, de acordo, com as características descritas na literatura [7,3]. A Tabela 1 apresenta a quantidade de artigos que estavam caracterizados corretamente em comparação à visão desta pesquisa. Dos 17 trabalhos, 3 não continham uma autodenominação quanto ao tipo de revisão. Das demais, 4 SLMs e 5 SLRs utilizavam nomenclatura condizente com a estrutura da pesquisa aplicada.

\begin{tabular}{|l|c|c|}
\multirow{2}{*}{$\begin{array}{c}\text { Visão dos própri- } \\
\text { os autores }\end{array}$} & $\begin{array}{c}\text { Visão dos autores } \\
\text { desta pesquisa }\end{array}$ & Avaliação \\
\hline \multirow{2}{*}{ SLM $(\mathrm{n}=5)$} & $\mathrm{SLM}=4$ & Correto \\
\cline { 2 - 3 } & $\mathrm{SLR}=1$ & Errado \\
\hline \multirow{2}{*}{$\mathrm{SLR}(\mathrm{n}=9)$} & $\mathrm{SLM}=4$ & Errado \\
\cline { 2 - 3 } & $\mathrm{SLR}=5$ & - \\
\hline \multirow{2}{*}{$\begin{array}{l}\text { Não identificaram } \\
\text { (n }=3)\end{array}$} & $\mathrm{SLM}=2$ & - \\
\cline { 2 - 3 } & $\mathrm{SLR}=1$ & Correto \\
\hline
\end{tabular}

Tabela 1: Comparação da autodenominação dos artigos e classificação adotada nesta pesquisa

A Figura 9 mostra os dados da Tabela 1 de forma sumarizada, sendo considerados apenas os artigos que apresentaram uma autodenominação para seu trabalho.

Dentre todos os 14 artigos apresentados na Figura 9, ressalta-se que, somente nove classificaram-se de forma condizente com as características descritas na seção 2 .

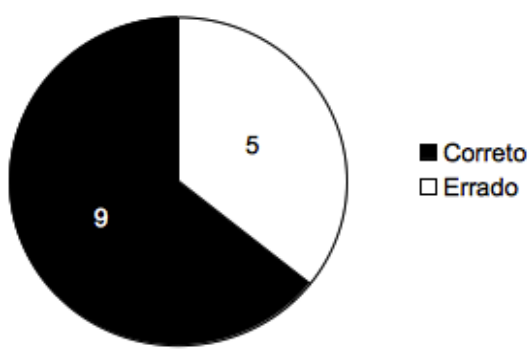

Figura 9: Quantidade de artigos autodenominados corretamente, de acordo com a visão desta pesquisa.

\section{Discussão}

O número de artigos de $\mathrm{PBs}$ vêm crescendo na última década mas, o aumento foi mais acentuado nos poucos últimos anos. Somente um dos veículos consultados não 
apresentou nenhum artigo de PB de nenhum tipo. Em todos os demais houve uma presença de cerca de $2 \%$ de artigos dessa natureza. O tipo de PB predominante ainda é o tradicional, porém já há um indício de pesquisas que se preocupam com a utilização de um método sistemático. Pesquisas que defendem a utilização de SLM e SLR (como [7], [3] e [25]) podem ter influenciado a popularização destes modelos na área de Computação. 42 de 3149 artigos sobre PB na área de Informática na Educação é pouco, mas percebe-se uma nítida tendência para o aumento do número de publicações, em especial artigos de revisão tendo estes se concentrado nos anos de 2011 e 2012.

A partir dos dados obtidos é possível observar que a falta de padronização na caracterização e denominação dos estudos dificulta a correta indexação dos trabalhos e que os mesmos sejam encontrados. Durante as buscas, cerca de 17 denominações diferentes foram usadas para diferenciar os 4 tipos considerados. Alguns artigos nem seriam descobertos numa busca, pela falta de padronização da terminologia de PB ou ausência desta. Consequentemente, este fato dificulta a realização de estudos secundários e, principalmente, terciários.

Observou-se que não há um padrão adotado na denominação dos trabalhos, sendo que o termo utilizado com maior frequência nesta amostragem foi "revisão", mesmo que alguns tenham sido usados erroneamente. Além disto, observa-se que 9 do total de 17 artigos avaliados nesta pesquisa denominaram-se como SLM ou SLR, porém destes 4 utilizavam a denominação incorretamente, ou seja, definiam-se como SLR e executaram um mapeamento/meta-análise ou vice-versa. Parte destes não citaram pesquisas reconhecidas como referências na aplicação destes métodos, tanto na área da Computação ou de outra Ciência como, por exemplo, os autores [3,7, 25]. Isto demonstra que ainda há confusão quanto aos termos e tipos de PB.

Identificou-se que, apesar de todas as 17 PB sistemáticas terem sido executadas por mais de um pesquisador, apenas 2 adotaram ou descreveram os critérios utilizados para resolução de conflitos na seleção dos artigos ou aplicação dos critérios de inclusão e exclusão. Esta informação é importante para que o processo possa ser replicado por outros pesquisadores e garantir que os resultados obtidos sejam imparciais.

Também foi observada em algumas das PBs, a presença de características tanto de SLM quanto de SLR, como a pesquisa apresentada neste presente texto. Isto mostra que muitas vezes seus métodos se complementam, sendo possível e necessário para responder as questões da pesquisa tanto identificar as tendências representadas pelas principais evidências encontradas através do estudo realizado, como apresentar em maior profundidade os conceitos e resultados obtidos pelas pesquisas avaliadas. De forma geral, os SLRs apresentaram sínteses narrativas dos trabalhos avaliados e os SLMs utilizaram-se de meta-análises, ou seja, sínteses quantitativas dos dados, demonstrando as evidências através de tabelas ou métodos estatísticos.

Apesar dos trabalhos selecionados apresentarem processos sistemáticos, alguns descrevem ou executam-no de forma superficial, sem detalhar o processo completo, dificultando assim a reprodução dos resultados por outros pesquisadores. Um dos artigos não apresentou as palavras-chaves utilizadas em sua estratégia de busca, dois deles não definiram de forma explícita as perguntas da pesquisa, um artigo não apresentou as bases ou ferramentas de busca utilizadas. Do total de 17 PBs sistemáticas, 4 não descreveram nenhum dos critérios de inclusão ou exclusão adotados na seleção dos estudos primários.

Estes dados sugerem que, na área de Informática na Educação, a condução de pesquisas bibliográficas sistemáticas ainda apresenta pouca maturidade, havendo a necessidade de maior disseminação dos métodos em sua execução.

\section{Conclusão}

Este artigo investigou o uso da Pesquisa Bibliográfica (PB) na área de Informática na Educação (IE) uma vez que este processo tem impacto direto na qualidade de uma pesquisa. Especial atenção foi dada para as PB sistemáticas por apresentarem elementos mais próximos ao rigor científico. Pôde-se observar uma aumento e tendência de crescimento do número de artigos sobre $\mathrm{PB}$, apesar de ainda ser uma pequena fração do total, menos que $2 \%$, mas já reflete a importância que a comunidade científica de IE está dando a este processo. Entretanto, observou-se que há confusão quanto a terminologia.

Através do mapeamento sistemático da literatura (SLM) realizado por esta pesquisa (detalhado ao longo do texto) foi possível observar que as PBs sistemáticas conduzidas na área de IE ainda necessitam de maior rigor na aplicação dos métodos e descrição do processo adotado. A utilização dos protocolos propostos por autores como [7], [3] e [25] podem auxiliar na execução das PBs. A importância do processo sistemático justifica-se pelo fato de que a aplicação de métodos científicos e sistemáticos demonstram um amadurecimento da área que os adotam. Como foi o caso encontrado na literatura relacionada, para a área da Engenharia de Software.

Utilizou-se na condução deste trabalho o protocolo de SLM proposto por [3], porém incluiu-se aqui uma síntese narrativa dos artigos avaliados devido a relevância dos mesmos. Desta forma, foi possível responder às perguntas de pesquisa da seguinte forma: 
- RQ1: Na amostra avaliada, foram identificados trabalhos de quatro tipos de PB: SLM, revisão sistemática da literatura (SLR), tradicional quanto bibliométrica, com predominância da tradicional;

- RQ2: Das 14 pesquisas que incluíram uma autodenominação, somente 9 utilizaram classificação condizente com as definições encontradas na literatura (apresentadas na seção 2);

- RQ3: De forma geral, as PBs sistemáticas estão utilizando os elementos do protocolo de revisão, porém dos 8 elementos analisados, apenas 2 foram encontrados em todos os trabalhos.

Os focos das pesquisas sistemáticas encontradas foram bastante variados, sendo todos diferentes entre si. Entretanto, pode-se mencionar que 4 deles envolviam aspectos relativos ao desenvolvimento de software educacional; 2 sobre uso de jogos na educação; 2 relacionavam-se ao ensino na área de computação e; 2 relacionamse com Ambientes Virtuais de Aprendizagem.

Esta pesquisa identificou ainda, que há uma dispersão dos trabalhos produzidos pela comunidade de IE em diversos sites diferentes o que dificulta e compromete a própria evolução da área. Também, reunir num único repositório os artigos dos veículos relacionados à área com recurso de busca pode facilitar a disseminação das pesquisas da área e tirar da obscuridade um amplo conjunto de experiências e inovações na área de IE, beneficiando a própria área como um todo. Iniciativas como o site da Comissão Especial de Informática na Educação (CEIE: www.br-ie.org) são louváveis pois ajudam muito, mas recursos de busca na base, ainda ausentes, se fazem

\section{Referências}

[1] R. S. Wazlawick. Uma reflexão sobre a pesquisa em ciência da computação à luz da classificação das ciências e do método científico. Revista de Sistemas de Informação da FSMA, 2010, 6:3-10.

[2] E. M. Lakatos, M. de A. Marconi. Metodologia Científica, 5 ed., Atlas, São Paulo, 1991, páginas 43-44.

[3] K. F. Petersen, S. Mujtaba, M. Mattsson. Systematic mapping studies in software engineering. In: Proceedings of the 12th international conference on Evaluation and Assessment in Software Engineering, EASE’08, páginas 68-77, 2008.

[4] S. R. Freeman, H. C. Williams, R. P. Dellavalle, C. Hewitt. The increasing importance of systematic reviews in clinical dermatology research and mais que necessários.

Também foi identificado que a comunidade está valorizando e usando cada vez mais as PB sistemáticas em várias áreas distintas, mas não adotou ainda todo o rigor científico que este mecanismo requer e nem uma terminologia uniformizada e clara. Assim, este artigo sugere que uma maior capacitação quanto à $\mathrm{PB}$ possa repercutir no aumento da qualidade científica dos trabalhos na área de Informática na Educação.

Como trabalhos futuros sugere-se realizar uma nova avaliação dos trabalhos selecionados através desta revisão, a fim de identificar se estes executam alguma atividade que não faça parte dos processos sistemáticos aplicados usualmente na área da Computação, como os descritos por $[3,7]$ ou atividades que invalidariam a sistematização da pesquisa e, consequentemente, sua replicação. Ainda, refazer este mapeamento dentro de 5 anos pode mostrar se houve alguma mudança nas tendências levantadas no presente texto.

\section{Agradecimentos}

Gostaríamos de agradecer aos colegas do curso de Mestrado em Computação Aplicada da Universidade do Estado de Santa Catarina, Adriano Pessini, Claudinei Dias, Franklin Gonçalves Jr., Helder Cognaco de Oliveira e Wilcilene Maria Kowal Schratzenstaller pelas contribuições na análise de alguns artigos.

publication. Journal of investigative dermatology, 126(11):2357-2360, 2006.

[5] A. J. Sutton, J. Higgins. Recent developments in meta-analysis. Statistics in medicine, 27(5):625650, 2008.

[6] D. S. Cruzes, T. Dybå. Synthesizing evidence in software engineering research. In: Proceedings of the 2010 ACM-IEEE, International Symposium on Empirical Software Engineering and Measurement, ESEM '10, páginas 1-10, 2010.

[7] B. A. Kitchenham, S. Charters. Guidelines for performing Systematic Literature Reviews in Software Engineering. Technical Report EBSE01, Keele University, 2007.

[8] J. A. Valente, F. J. de Almeida. Visão analítica da informática na educação no Brasil: a questão da formação do professor. Revista Brasileira de 
Informática na Educação, 1(1): 45-60, 1997.

[9] B. A. Kitchenham, P. Brereton, M. Turner, M. Niazi, S. Linkman, R. Pretorius, D. Budgen. The impact of limited search procedures for systematic literature reviews - a participant-observer case study. In: 3rd International Symposium on Empirical Software Engineering and Measurement, páginas 336-345, 2009.

[10] M. Nasser, V. Welch, P. Tugwell, E. Ueffing, J. Doyle, E. Waters. Ensuring relevance for Cochrane reviews: evaluating processes and methods for prioritizing topics for cochrane reviews. Journal of Clinical Epidemiology, 66(5): 474-482, 2013.

[11] B. A. Kitchenham, T. Dybå, M. Jorgensen. Evidence-based software engineering. In: Proceedings 26th International Conference on Software Engineering - ICSE, páginas 273-281, 2004

[12] A. Pritchard. Statistical bibliography or bibliometrics? Journal of Documentation, 4(25):348-349, 1969.

[13] B. A. Kitchenham, O. P. Brereton, D. Budgen, M. Turner, J. Bailey, S. Linkman. Systematic literature reviews in software engineering-a systematic literature review. Information and software technology 51(1 ): 7-15, 2009.

[14] G. K. Hanssen, D. Smite, N. B. Moe. Signs of agile trends in global software engineering research: A tertiary study. In: Sixth IEEE International Conference on Global Software Engineering Workshop (ICGSEW), 17-23, 2011

[15] H. Zhang, M. A. Babar. Systematic Reviews in Software Engineering: An Empirical Investigation, Information and Software Technology, 2012.

[16] S. Imtiaz, M. Bano, N. Ikram, M. Niazi. A tertiary study: experiences of conducting systematic literature reviews in software engineering,. In: 17th International Conference on Evaluation and Assessment in Software Engineering (ACM), 177-182, 2013.

[17] F. Q. B. da Silva, A. L. M. Santos, S. Soares, A. C. C. França, C. V. F. Monteiro, F. F. Maciel. Six years of systematic literature reviews in software engineering: An updated tertiary study. Information and Software Technology, 53(9): 899-913, 2011.

[18] A. C. Barros, J. Wainer, K. Claudio, L. R. B. Ferreira, T. Dwyer. Uso de computadores no ensino fundamental e médio e seus resultados empíricos: uma revisão sistemática da literatura. Revista Brasileira de Informática na Educação, 16(01): 57-68, 2008.

[19] L. F. Maximo, D. A. C. Barone, M. J. S. Carvalho. Informática e avaliação na educação a distância na UFRGS: um panorama de 1998 a 2008. Revista Novas Tecnologias na Educação (RENOTE), 6(1):1-10, 2008.

[20] W. Igarashi, D. C. C. Igarashi, M. K. Nakayama. Análise do alinhamento entre os elementos de aprendizagem organizacional/ gestão do conhecimento/ tecnologia da informação no contexto nacional e internacional. Revista Novas Tecnologias na Educação (RENOTE), 7(1):1-9, 2009.

[21] M. L. Löbler, L. M. Bolzan, F. I. Kubota, S. A. M. Flores, T. G. F. Kich. Inclusão Digital: Mapeamento de Publicações sobre o Tema, na Área de Administração. Revista Novas Tecnologias na Educação (RENOTE), 9(3):1-11, 2011.

[22] M. H. Pietruchinski, J. C. Neto, A. Malucelli, S. Reinehr. Os jogos educativos no contexto do SBIE: uma revisão sistemática de Literatura. In: Anais do Simpósio Brasileiro de Informática na Educação, páginas 476-485, 2011.

[23] J. Valaski, A. Malucelli, S. Reinehr. Revisão dos Modelos de Estilos de Aprendizagem Aplicados à Adaptação e Personalização dos Materiais de Aprendizagem. In: Anais do Simpósio Brasileiro de Informática na Educação, páginas 844-847, 2011.

[24] T. N. Bogoni, M. S. Pinho. Uma Revisão Sistemática de Simuladores de Endodontia Equipados com Dispositivos Hápticos. In: Anais do Simpósio Brasileiro de Informática na Educação, páginas 446-455, 2011.

[25] M. Pai, M. McCulloch, J. D. Gorman, N. Pai, W. Enanoria, G. Kennedy, P. Tharyan, J. M. Colford Jr. Systematic reviews and meta-analyses: an illustrated, step-by-step guide. The National Medical Journal of India, 17(2):86-95, 2004.

[26] F. Penteado, J. C. Gluz, C. Galafassi. Suporte Técnico/Pedagógico aos OAs pelos AVAs: uma Análise Comparativa das Pesquisas Recentes. In: Anais do Simpósio Brasileiro de Informática na Educação, páginas 294-303, 2011.

[27] V. C. O. Aureliano, P. C. de A. R. Tedesco. Ensino-aprendizagem de Programação para Iniciantes: uma Revisão Sistemática da Literatura focada no SBIE e WIE. In: Anais do Simpósio Brasileiro de Informática na Educação, páginas 1-10, 
2012.

[28] N. F. D. Filho, E. F. Barbosa. Estudo e Definição de um Conjunto de Características e Requisitos para Ambientes de Aprendizagem Móvel. In: Anais do Simpósio Brasileiro de Informática na Educação, páginas 1-10, 2012.

[29] F. Abreu, A. Almeida, E. Barreiros, J. Saraiva, S. Soares, A. Araújo, G. Henrique. Métodos, Técnicas e Ferramentas para o Desenvolvimento de Software Educacional: Um Mapeamento Sistemático. In: Anais do Simpósio Brasileiro de Informática na Educação, páginas 1-10, 2012.

[30] F. P. A. de Medeiros, A. S. Gomes. Monitoramento da Experiência do Usuário em Ambientes Colaborativos Virtuais de Aprendizagem: Um Mapeamento Sistemático. In: Anais do Simpósio Brasileiro de Informática na Educação, páginas 1-10, 2012.

[31] S. C. Vermelho, A. P. M. Velho, A. R. Pirola, A. K. Bonkovosky. Perfil das pesquisas e publicações sobre Promoção da Saúde e Redes Sociais no Brasil. In: Anais do Simpósio Brasileiro de Informática na Educação, páginas 1-5, 2012.

[32] M. M. de Lima, A. R. de Lima, A. C. C. Monteiro, E. H. Cavalcante Jr, L. de Q. L. Gomes. Uma Revisão Sistemática da Literatura dos Processos de Desenvolvimento de Software Educativo. In: Anais do Simpósio Brasileiro de Informática na Educação, páginas 1-5, 2012.

[33] L. Paludo, A. Raabe. Análise de Jogos Educativos de Computador para Gerência de Projetos de Software. In: Workshop sobre Educação em Computação, páginas 867-876, 2010.

[34] F. B. V. Benetti, E. L. Albano. Teste de Software: o que e como é ensinado? In: Workshop sobre Educação em Computação, páginas 1-10, 2012.

[35] A. M. C. de A. Oliveira, R. L. Rodrigues, V. C. Garcia. Um Mapeamento Sistemático para Problem Based Learning aplicado à Ciência da Computação. In: Workshop de Informática na Escola, páginas 1-10, 2012. 